\title{
MATE SELECTION PREFERENCES IN GERMANY AND THE UNITED STATES
}

\author{
David M. Buss ${ }^{1 *}$ and Alois ANGleitneR ${ }^{2}$ \\ ${ }^{1}$ University of Michigan, Ann Arbor, MI 48109-1346, U.S.A. and ${ }^{2}$ University of Bielefeld, \\ 4800 Bielefeld, F.R.G.
}

(Received 6 February 1989)

\begin{abstract}
Summary-Two studies examined expressed mate selection preferences in German and American samples. In the first study (German $N=343$; American $N=313$ ), subjects ranked 13 characteristics on their desirability in a potential mate. Large and consistent sex differences were predicted and found within each country on valuation of good earning capacity (females more) and physical attractiveness (males more). The largest cultural differences were found for valuation of the characteristics Good Housekeeper (Germans more) and Physical Attractiveness (Americans more). A second study (German $\mathrm{N}=751$; American $\mathrm{N}=1137$ ) was conducted to replicate and extend these results using two separate testing instruments and larger more diverse samples within each country. The basic sex differences within countries and cultural differences across sexes were robustly replicated. None the less, the two countrics showed remarkable similarity in patterns of mating preferences across characteristics. Discussion emphasizes the theoretical significance of these findings and identifies important directions for future research in human mating systems.
\end{abstract}

Human mating patterns are intriguing at least in part because they are caused by, and have consequences for, so many scientific levels of analysis. Sociologists are concerned about the effects of educational institutions on mate selection, and about the effects of mate selection on the distribution of wealth. Evolutionary biologists are concerned about the effects of nonrandom mating on directional selection; geneticists are concerned about the consequences for changes in the distribution of genotypes within and between families over generations (Buss, 1985; Crow and Kimura, 1970; Eckland, 1968; Jensen, 1978; Vandenberg, 1972).

The psychological study of mate selection lies between the biological and sociological, and has implications for both levels. One of the most important psychological questions about human mating is: What criteria do individuals use in selecting a mate? In mating systems where individual choice is exerted, psychological preferences may be expected to provide an important, although not the only, determinant of mating decisions (Buss and Barnes, 1986).

Mate selection criteria have been studied intermittently within the United States (e.g. Burgess and Wallin, 1953; Buss, 1985; Elder, 1969; Hill, 1945; Hudson and Henze, 1969; Langhorne and Secord, 1955; McGinnis, 1958; Rushton, 1984). Some cross-national research has examined such phenomena as attitudes toward sexual permissiveness (Raschke and $\mathrm{Li}, 1979$ ), attitudes toward marriage (Podmore and Chaney, 1979), and attitudes toward marital infidelity (Christensen, 1979). Few studies, however, have directly examined mate selection criteria in different countries (cf. Buss, 1989; Murstein, 1979).

The broad purpose of the present studies was to identify the similarities and differences in mate selection criteria in West Germany and the United States. Within the context of this broad purpose, we sought to identify (1) similarities and differences between German and American males in their mate preferences; (2) similarities and differences between German and American females in their mate selection preferences, (3) the cross-cultural generality of two clusters of sex differences in mate selection criteria that have been found repeatedly within the United States, (4) the effects of age, family size, and other variables on mate preferences in both countries, and (5) the generalizability of the above results across two different methods of obtaining mate selection criteria.

*To whom all correspondence should be addressed. 


\section{Guiding methodological principles}

Three methodological principles guided our research. First, we sought to obtain large samples from each country so that obtained results would be generalizable and not be subject to the vagaries of small samples. Second, we adopted two separate methods for obtaining expressed mate selection criteria so that we could examine the generalizability of the results across methods. And third, we imposed conservative statistical criteria for identifying and discussing major results.

\section{Hypothesized sex differences}

Two major clusters of sex differences in mate selection criteria have been found within the United States (Buss, 1985, 1987; Buss and Barnes, 1986). The first is that females, more than males, value the cluster of characteristics surrounding good earning potential. This includes not only income per se, but also the characteristics that tend to covary with good income: ambition, industriousness, professional degrees, older age, and good social status.

The second cluster of sex differences repeatedly found within United States samples is that males, more than females, value physical attractiveness, good looks, and youth in potential mates (Buss, 1985; Buss and Barnes, 1986; Berscheid and Walster, 1974; Langhorne and Secord, 1955; McGinnis, 1958; Hudson and Henze, 1969).

Two hypotheses have been advanced to account for these sex differences (Buss and Barnes, 1986): (1) the structural powerless and sex-role socialization hypothesis, and (2) an evolutionary hypothesis based on cues to reproductive investment. The first hypothesis starts with the assumption that females are typically excluded from the power structure and viewed as objects of exchange. Because of imposed restrictions on individual advancement, females seek in mates those characteristics associated with power such as earning capacity and higher education. In contrast, males place a premium on the 'exchange object' itself, and so value physical attractiveness in potential mates more than do females (enhanced value as a sex object). Traditional sex-role socialization practices are assumed to maintain and support these structural differences, and are used to inculcate role-appropriate values in males and females.

The second hypothesis is based on evolutionary theory (Darwin, 1871; Fisher, 1958; Trivers, 1972). This hypothesis states that males and females will both seek in mates qualities that will best increase their reproductive success (gene replication). Because of sex differences in the constraints on reproductive success, males and females will value different characteristics. Specifically, because females invest more heavily in fewer offspring and have sharp constraints on quantity, the primary reproductive constraint on females centers around social and material provisions for their fewer offspring. Females therefore will value mate characteristics associated with earning capacity such as ambition, industriousness, status, professional degrees and older age.

In contrast, the primary constraint on a male's reproductive success is access to fecund and reproductively valuable females. Since relative youth and physical appearance provide strong cues to fecundity and reproductive value in females, males are hypothesized to value them in potential mates. Standards of female beauty are hypothesized to have evolved to correspond to relative reproductive value (expected number of future offspring) or fertility (probability of current conception). Since male appearance is not strongly correlated with resource potential, females are hypothesized to value appearance less than males.

The two hypotheses are not intrinsically incompatible, but they differ in three respects. One major difference is that the evolutionary hypothesis specifies an account of the ultimate origins of the sex differences, and indeed of the origins of male control over resources generally. In this account, males strive to control resources because females value resource acquisition in potential mates; hence, males with resources enjoy preferential mating opportunities. The 'structural powerlessness' hypothesis leaves unspecified the ultimate causes, and deals more specifically with maintenance of the status quo.

A second difference between the two hypothesis is that the structural powerless hypothesis specifies the causal path by which the sex differences are produced, namely socialization practices. In contrast, the evolutionary hypothesis leaves unspecified the causal paths. In principle, the parental socialization could be the causal path by which the evolutionary hypothesis operates. Alternatively, genetic differences between males and females in the bases of attraction could be the causal path. A third possibility is that both paths could operate in concert. 
A third difference between the hypotheses is that the structural powerlessness hypothesis predicts large cross-cultural differences in the existence of these sex differences. From the evolutionary hypothesis, one would also predict that ecological conditions might affect the strength of the sex differences. For example, in cultures where there is little variance in male resource holdings, females should place less emphasis on this characteristic because the ultimate reproductive advantages of seeking this characteristic would not be as great. For such cultures, little sex difference would be predicted. However, male valuation of female youth and appearance should be highly generalizable across cultures because they provide the strongest available cues to the reproductive value of a female. Thus, cross-cultural data is urgently needed to address these issues.

\section{STUDY I}

\section{Method}

German sample. The German sample was composed of students attending the University of Bielefeld and the University of Duesseldorf. The sample of 343 Germans consisted of 174 males and 169 females.

American sample. The American sample was composed of students attending the University of California at Berkeley and Harvard University. The sample of 313 Americans consisted of 106 males and 207 females.

Factors in mate selection questionnaire. This instrument was based on previous factor analyses of a larger 76-item instrument (Buss and Barnes, 1986) initially developed by Gough (1973). The highest loading items from each factor were represented, as were several additional items for testing the specific hypotheses about sex differences. In addition to indicating their age and sex, $S$ s read the following instructional set:

\footnotetext{
"Below are listed a set of characteristics that might be present in a potential mate or marriage partner. Please rank them on their desirability in someone you might marry. Give a ' $l$ ' to the most desirable characteristics in a potential mate; a ' 2 ' to the second most desirable characteristic in a potential mate; a ' 3 ' to the third most desirable characteristic; and so on down to '13' for the 13th most desired characteristic in a potential mate."
}

Following the instructional set, each $S$ ranked the 13 mate characteristics from most (1) to least (13) desired in a potential mate.

German translation. A bilingual professional translator (a native German residing in the United States) was employed to translate the English version of the questionnaire into German. Special instructions were provided to ensure that the items were 'sex-neutral'. In English, for example, the term 'physically attractive' may be used to describe either males or females, but the words 'handsome' or 'beautiful' would be sex-biased in the sense of being more applicable to one sex than to another. The translated version was back-translated by another bilingual speaker, and discrepancies were resolved by a third bilingual speaker.

\section{Results}

\section{Background characteristics}

The mean ages of the German samples were $24.40 \mathrm{yr}$ for males and $25.26 \mathrm{yr}$ for females. The corresponding mean ages for the American samples were 22.23 and 23.95 . Approximately $12 \%$ of the German males were married, while approx. $16 \%$ of the German females were married. In the American sample, these figures were 11 and $13 \%$.

\section{MANOVA tests for overall national and sex differences}

Because of the multivariate nature of this study, we conducted a $2 \times 2 \times 2$ MANOVA, entering country (Germany, United States) and sex (male, female) as independent variables and the set of ranked preferences as dependent variables excluding the last variable, health. (In MANOVA's conducted on ranked variables, one variable must be excluded from the analysis to meet statistical assumptions.)

The effect of country was highly significant $(F=20.84$, d.f. $=631, P<0.0001)$. Similarly, the effect of sex was highly significant $(F=20.08$, d.f. $=631, P<0.0001)$. The significance of this test 
suggested that there existed national and sex differences in the mate preference rankings that could be examined in more detail. The interaction between country and sex was not significant $(F=1.09$, d.f. $=12, P=0.363$ ).

Sex differences cross-culturally. $t$-Tests were conducted to test for sex differences for each item for each country separately. These results are shown in Table 1. In order to interpret these results, a conservative statistical criterion was used. Specifically, a result was required to be significant beyond the 0.001 level (2-tailed) before interpretation.

As shown in Table 1, females in both the German and American samples ranked good earning capacity significantly higher than did their male counterparts. In contrast, males in both samples ranked physically attractive higher than their female counterparts. No other characteristic passed our statistical criterion in both cultures.

Cross-cultural similarities and differences. To examine where the national differences resided, $t$-tests were conducted for each item, one for German and American males and one for German and American females. These results are also shown in Table 1.

Using the same conservative statistical criterion (significance beyond the 0.001 level), the largest cultural difference occurred for the characteristic good housekeeper. Both German males and females valued this characteristic in a potential mate more than did American males and females. Americans valued physical attractiveness and college graduate in mates significantly more than did Germans.

In order to evaluate the overall similarity between the German and American mate preferences, $\rho$ correlations were computed across the means for the 13 characteristics, 1 for each sex. The $\rho$ correlation between German and American males was $0.91 \quad(P<0.001)$. The corresponding coefficient for females was $0.91(P<0.001)$. These results suggest that, in spite of significant and meaningful differences in the mate values of the two countries, there exists a tremendous similarity in the overall patterning of values.

In sum, several large differences were found between German and American samples. These centered on valuation of good housekeeper (Germans more), and physical attractiveness, and college graduate (Americans more). In spite of these clear differences, the two cultures are overwhelmingly similar in the mean ranks across the 13 characteristics.

\section{STUDY II}

In the second study, we sought to replicate and extend the results of the first study. Specifically, we sought to obtain larger and more heterogeneous samples of $S$ s within each country. Second, we included a second instrument to supplement the ranking procedure in an attempt to transcend method specificity.

\section{Method}

German sample. The German sample was composed of a mixture of students from the University of Bielefeld, the University of Dusseldorf, and resident populations in the surrounding regions. Ss were recruited through local newspapers. The sample of 751 Germans consisted of 363 males and 388 females.

American sample. The American sample consisted of students from four different universities: University of Michigan, University of Texas, University of California, and Harvard University. Ss were recruited through classes. The sample of 1137 consisted of 512 males and 625 females.

Procedures. Ss completed two instruments. The first was the Factors in Mate Selection questionnaire described in Study I. The second was an instrument designed within the United States in the 1930s to assess preferences in mate selection (Hill, 1945). This instrument consisted of two parts: (1) background information (e.g. age, sex, religion, marital status, number of brothers, number of sisters, number of children wanted, age at which marriage is preferred, age difference preferred between self and spouse, and preference for who should be older), (2) evaluation of 18 characteristics on how desirable they are in a potential mate.

The evaluation portion of the instrument was different in format from the first procedure in that $S$ s were not forced to rank the characteristics. Instead, they rated each characteristic on a 4-point scale: 3 = indispensable; 2 = important, but not indispensable; $1=$ desirable, but not very important; $0=$ irrelevant or unimportant. 
Results

Background characteristics. Table 2 shows the background characteristics of the two samples on age, marital status, number of brothers and sisters, age at which marriage is preferred, age difference preferred between spouses, who is preferred to be older, and how many children are desired. The first two rows in Table 2 show clearly that the German and American samples differ substantially in age and marital status. The German sample is older on average. A higher percentage of the German sample is married. And perhaps as a function of their greater age, the Germans in this sample prefer to be married approx. 3 yr later than their American counterparts. These sample differences present simultaneously a weakness and a strength of this replication study. The weakness consists of the fact that the samples are not strictly comparable. The strength lies in the increased generality of the findings if they corroborate those of the first study. In addition, the diversity in age and marital status permits examination of the effects of these variables on mate selection preferences. These issues and findings are discussed in greater detail below.

In spite of these sample differences, the Germans and Americans are remarkably similar in whom is preferred to be older. In both samples, $87 \%$ of the males prefer to be older than the person that they marry. Among the females, $94 \%$ of the Germans and $98 \%$ of the Americans prefer that the person that they marry be older. The magnitude of the preferred difference, however, does differ for Germans and Americans, perhaps as a function of the age differences. Overall, Germans prefer a greater age difference than their American counterparts in these samples.

Finally, the females of the two countries do not differ in how many children are desired. The American males, however, desire slightly more children than their German counterparts.

$M A N O V A$ tests on rankings and ratings. As in the first study, MANOVA analyses were conducted to test for overall national and sex differences. These MANOVA's showed highly significant main effects for both nationality and for sex. For the ranking instrument, the main effect for nationality was $F=31.92$ (d.f. $-1077, P<0.0001$ ) and for sex was $F=48.59$ (d.f. $=1077$, $P<0.0001$ ). For the rating procedure, the main effect for nationality was $F=63.45$ (d.f. $=1804$, $P<0.0001$ ) and for sex was $F=67.98$ (d.f. $=1804, P<0.0001$ ).

Sex differences cross-culturally. Table 3 shows the results of the replication on the larger samples of Germans and Americans. The basic sex differences in valuation of good earning capacity and physical attractiveness are robustly replicated within each of the two cultures. Indeed, like the first study, these two characteristics show the largest magnitude of sex differences for within each country. German females place greater value than German males on Kind-Understanding and Intelligent. American females place greater value on Health than do American males. The greater female preference for Kind-Understanding mates is replicated only in the German sample. Finally, within both samples, males value in a potential mate Good Housekeeper significantly more than do females.

Cross-cultural differences and similarities. Also shown in Table 3 are the $t$-tests on GermanAmerican differences for males and females separately. As in the first study, Good Housekeeper is given greater value by German $S$ s than by American $S$ s. Similarly, Germans more than Americans place greater value on the mate characteristic of Easygoing-a finding that replicates that of the first study. Finally, as in the first study, Americans place greater value than Germans on Physical Attractiveness in a potential mate. In sum, although small differences exist between Study I and Study II, the major sex differences across cultures and the major cultural differences across sex are robustly replicated.

$\rho$ Correlation coefficients were computed to determine the overall pattern similarity between the two countries for Study II. For males, the correlation is 0.96 across the 13 characteristics, while for females, the correlation is 0.84 . These high magnitudes suggest again that, in spite of significant cultural differences, there is large pattern similarity in the values placed on different mate characteristics across the two cultures.

Mate preference ratings. Table 4 shows the analogous results for the 18 -item rating instrument. It should be noted in this context that there are advantages and drawbacks to the ranking and rating procedures. In general, the ranking procedure forces the distribution within each $S$ such that the total must sum to a constant number. This has the advantage of clarifying real discriminations that exist among the mate characteristics by preventing $S$ s from ranking them all high or all low. 

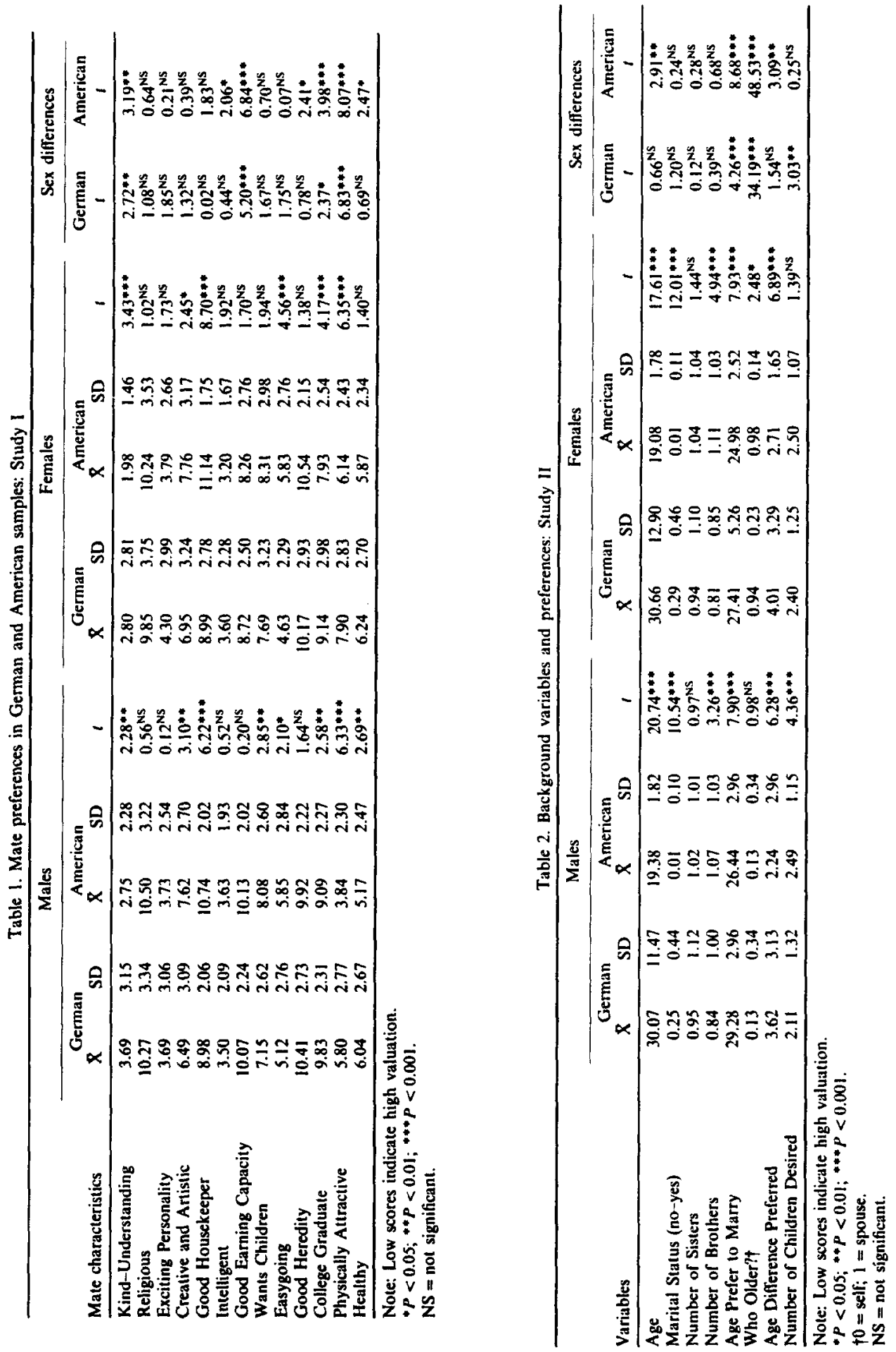

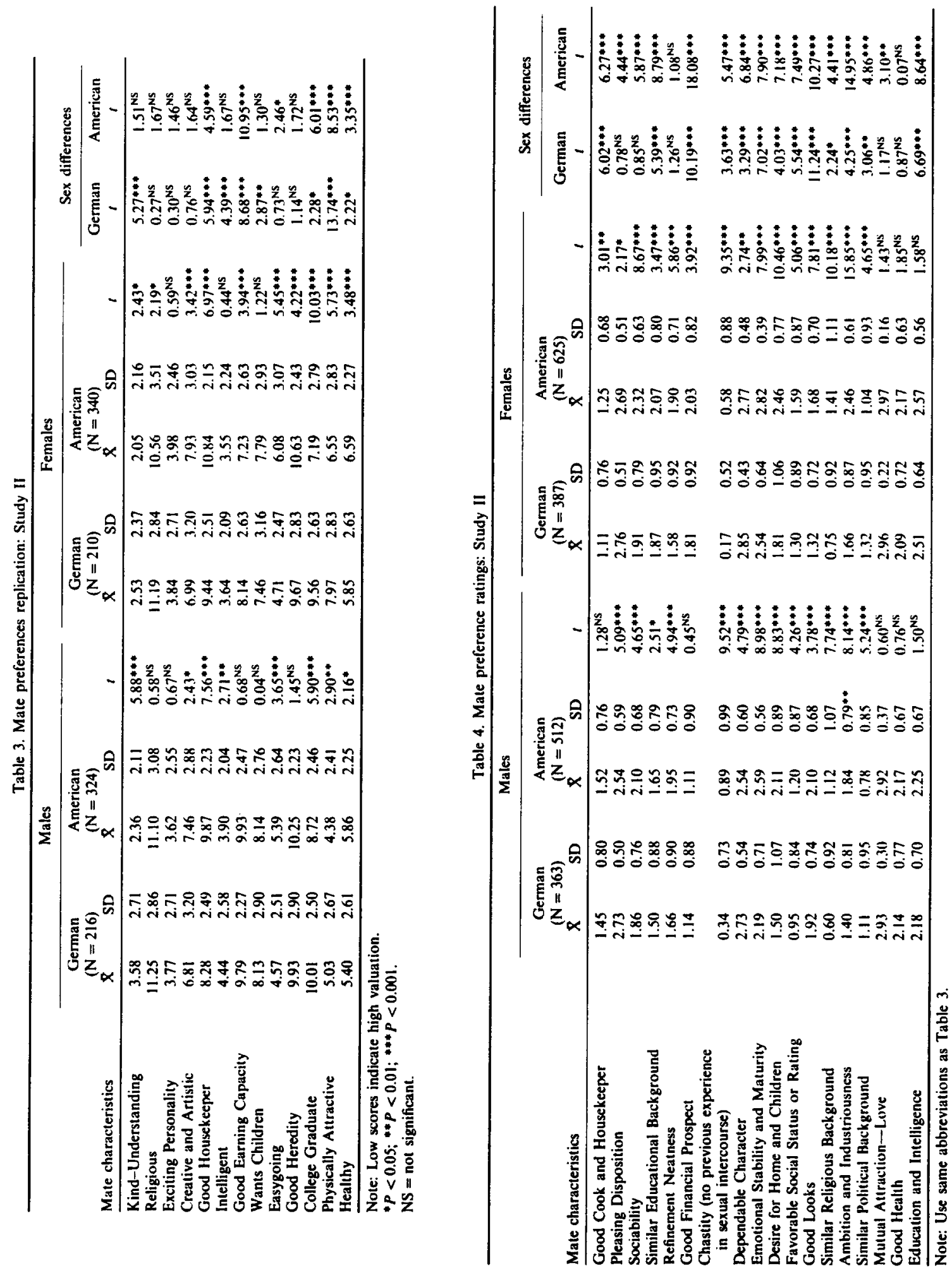
Table 5. Correlations between background

\begin{tabular}{|c|c|c|c|c|c|c|c|c|}
\hline \multirow[b]{3}{*}{ Mate characteristics } & \multicolumn{4}{|c|}{ Age } & \multicolumn{4}{|c|}{ Number of brothers } \\
\hline & \multicolumn{2}{|c|}{ German } & \multicolumn{2}{|c|}{ American } & \multicolumn{2}{|c|}{ German } & \multicolumn{2}{|c|}{ American } \\
\hline & $\mathbf{M}$ & $\mathbf{F}$ & $\mathrm{M}$ & $\mathrm{F}$ & $\mathbf{M}$ & $\mathrm{F}$ & $\mathbf{M}$ & F \\
\hline Kind-Understanding & $13^{*}$ & $13^{* *}$ & -01 & 03 & -03 & -01 & -04 & $-12 *$ \\
\hline Religious & 02 & 07 & -01 & 01 & -02 & -03 & 12 & 09 \\
\hline Exciting Personality & $-14 * \cdots$ & $-14 * *$ & $-15^{*}$ & $-14^{*}$ & 04 & -07 & -03 & -08 \\
\hline Creative and Artistic & $-22 * * *$ & $-33 * \cdots *$ & 05 & 03 & 04 & -01 & 06 & -04 \\
\hline Good Housekeeping & $31 * * *$ & 00 & $15^{*}$ & 09 & -06 & -02 & 02 & -02 \\
\hline Intelligent & $-13 * *$ & 03 & -08 & 01 & 06 & 03 & -01 & $-19 * *$ \\
\hline Good Earning Capacity & -06 & $21 * * *$ & -03 & -03 & $-10^{*}$ & 01 & -11 & 04 \\
\hline Wants Children & 06 & 05 & -02 & $-13^{\circ}$ & 07 & -02 & 06 & 07 \\
\hline Easygoing & 03 & 00 & 08 & -06 & -06 & 06 & 09 & 08 \\
\hline Good Heredity & $29 * * *$ & $37 * * *$ & $14^{*}$ & 08 & -05 & $11 *$ & 08 & 11 \\
\hline College Graduate & $-13 *$ & $-12 *$ & -08 & 06 & -05 & $-13 * *$ & -09 & -08 \\
\hline Physically attractive & $-20 * * *$ & $-35 * * *$ & -02 & -04 & -01 & -09 & -11 & 01 \\
\hline Healthy & 08 & $14 * *$ & -02 & $13^{*}$ & $15^{* *}$ & $14^{* * *}$ & -08 & 03 \\
\hline Age & - & - & - & - & & & & \\
\hline Number of Brothers & 04 & 03 & $09^{*}$ & -05 & - & - & - & - \\
\hline Number of Sisters & $14 * *$ & $12 * *$ & -01 & 07 & $17 * * *$ & 07 & 02 & -01 \\
\hline Age Prefer to Marry & $-15^{* *}$ & $-15^{* *}$ & 06 & 05 & -04 & -03 & 06 & -10 \\
\hline Number of Children Wanted & $13 * *$ & 07 & 01 & -04 & $25^{* * *}$ & $10^{*}$ & 01 & $28^{* * *}$ \\
\hline
\end{tabular}

* $P<0.05 ; * P<0.01 ; * * P<0.001$.

But this strength has a corresponding drawback - it precludes the discovery of overall elevation across characteristics because it forces the distribution.

In contrast, the rating procedure permits placement of many characteristics high or low, and thus can discover, for example, whether males or females are more choosy or exacting in their expressed preferences. Its drawback is a corresponding potential lack of discrimination among characteristics that do differ in their subjective value.

Since all measurement instruments contain sources of bias, our guiding rationale for Study II was to use two methods that differ in their potential biases. Results that emerge across both methods can be treated with greater confidence than results that emerge from a single method. Because of the partial overlap in characteristics for the two methods, we were able to example cross-method correlations at the individual level.

As shown in Table 4, the two largest sex differences within both the German and American samples are Good Financial Prospect (valued by females more) and Good Looks (valued by males more). That these sex differences are consistently the largest, across four samples from two countries using two separate methods, lends considerable credence to their robustness.

Across both countries, fernales more than males, appear to value Similar Educational Background, Emotional Maturity and Stability, Desire for Home and Children, Favorable Social Status, Ambition and Industriousness, and Education and Intelligence in a potential mate. In contrast, males in both countries more than females value Good Cook and Housekeeper, and Chastity in a potential mate, although overall valuation of Chastity is low in absolute terms, for both sexes.

The American sample, more than the German sample, placed greater value on Sociability, Refinement and Neatness, Chastity, Emotional Maturity and Stability, Desire for Home and Children, Favorable Social Status, Good Looks, Similar Religious Background, Ambition and Industriousness, and Similar Political Background. In contrast, Germans appear to place greater emphasis on Pleasing Disposition and Dependable Character more than their American counterparts. The large German preference for Good Housekeeper found in the ranking data from both studies, however, is not replicated using the rating procedure.

Correlations between background variables and mate preferences. Tables 5 and 6 show the correlations between certain background and preference variables and the ranked and rated mate preferences from Study II. Age shows several interesting and consistent correlates across samples and sexes. Exciting Personality covaries negatively with age in all four samples-older $S$ s apparently care less about having a mate with an exciting personality. Within the German sample, for which there is greater variance in age, age covaries negatively with preferences for Creative and Artistic, Physically Attractive, Good Looking, and College Degree. Also within the German sample, age covaries positively with desiring a mate who is Kind and Understanding, has Good Heredity, a Similar Educational Background, Dependable Character, Emotional Stability and 
variables and mate preferences: rankings

\begin{tabular}{|c|c|c|c|c|c|c|c|c|c|c|c|}
\hline \multicolumn{4}{|c|}{ Number of sisters } & \multicolumn{4}{|c|}{ Age prefer to marry } & \multicolumn{4}{|c|}{ Number of children wanted } \\
\hline \multicolumn{2}{|c|}{ German } & \multicolumn{2}{|c|}{ American } & \multicolumn{2}{|c|}{ German } & \multicolumn{2}{|c|}{ American } & \multicolumn{2}{|c|}{ German } & \multicolumn{2}{|c|}{ American } \\
\hline $\mathrm{M}$ & $F$ & $\mathbf{M}$ & $\mathbf{F}$ & $\mathbf{M}$ & $F$ & $\mathbf{M}$ & $\mathbf{F}$ & $\mathbf{M}$ & F & $\mathbf{M}$ & $\mathbf{F}$ \\
\hline-03 & 09 & 05 & 00 & $-05^{*}$ & $-13^{*}$ & -10 & 00 & 05 & 04 & -07 & 04 \\
\hline-01 & $13^{* *}$ & 00 & $12^{*}$ & -08 & $-14^{* *}$ & $-13^{*}$ & -05 & $11=$ & $18=0$ & 11 & $16^{* *}$ \\
\hline 02 & 00 & 06 & -04 & $10^{*}$ & $15^{* *}$ & 05 & -05 & 00 & $-11 *$ & -01 & -03 \\
\hline 04 & -02 & -01 & -03 & 06 & $22 * \cdots$ & 07 & $17^{* *}$ & $-12^{*}$ & $-13^{* *}$ & 04 & -10 \\
\hline$\infty$ & 02 & 03 & -02 & $-10^{*}$ & 06 & -10 & $13^{*}$ & 00 & $-10^{*}$ & -02 & $-19 * *$ \\
\hline $14^{* *}$ & $-16^{* *}$ & -07 & 04 & $11 *$ & $12^{*}$ & $19^{* *}$ & 11 & -08 & -09 & 09 & -08 \\
\hline-09 & -07 & $-17 * *$ & 06 & $18 * * *$ & $-10^{*}$ & 01 & -08 & $-19 * * *$ & -03 & $-19 * *$ & -06 \\
\hline-01 & 07 & -05 & -03 & $-21 * * *$ & $-23^{* * *}$ & $-32 * * *$ & $-27^{* * *}$ & $45^{* * *}$ & $41^{* * *}$ & $21 * \cdots$ & $35 * * *$ \\
\hline-01 & 09 & -08 & -08 & 00 & -06 & -02 & 03 & -03 & $-11^{*}$ & -10 & -07 \\
\hline-04 & 02 & 07 & -05 & $-12^{*}$ & -06 & $13^{*}$ & 06 & 05 & 03 & -03 & 04 \\
\hline-05 & $-09^{*}$ & -06 & 05 & 10 & 05 & $18^{* *}$ & 11 & $-27 * * *$ & -04 & $-12^{*}$ & 09 \\
\hline OI & $-15^{* *}$ & 08 & -07 & $14^{*}$ & $15^{* *}$ & 04 & -08 & $-14 *$ & $-13^{*}$ & 04 & $-14^{*}$ \\
\hline 04 & 03 & $17 * *$ & 07 & -07 & -03 & 07 & -01 & 11 & 00 & 00 & -10 \\
\hline - & - & - & - & & & & & & & & \\
\hline-05 & $-09^{*}$ & -02 & 03 & - & - & - & - & & & & \\
\hline $14^{* *}$ & 03 & $32 * * *$ & $07^{*}$ & $-11^{*}$ & $-27 * * *$ & $-09^{*}$ & $-18 * * *$ & - & - & - & - \\
\hline
\end{tabular}

Maturity, Desire for Home and Children, Favorable Social Status, Ambition and Industry, and Good Health.

In contrast, the family size variables (number of brothers and sisters) show few mate preference correlates that are consistent across nationality and sex. The only finding of note is that coming from a large family seems to go along with desiring a larger number of children. This is a potentially important finding, as it suggests that differences in family size may be correlated over generationsa finding of considerable interest to population geneticists and evolutionary biologists.

Age at which marriage is preferred has several consistent and interesting correlates with mate preferences. Specifically, those who desire to marry later in life appear not to want children as much as those who wish to marry earlier in life. Those preferring late marriage also care less about the chastity of their mate, and more about the intelligence of their mate than do those preferring to marry earlier. Among German males and females only, later age marriage preferences are also associated with a stronger preference for a mate who is physically attractive and has a similar political background.

Finally, what are the correlates of Number of Children Desired? Across nationality and sex, wanting a large number of children appears to be associated with preferring a mate who is Religious and who has a Similar Religious Background. Those desiring a large family also appear to want their mate to be chaste, and place less emphasis on physical attractiveness. Finally, as mentioned earlier, large family preference is associated with having a large family of origin and with a desire to marry early.

Cross-method checks. To examine the consistency of individual responses across the rating and ranking methods, we correlated individual scores for two pairs of variables: Physically Attractive (ranking) and Good Looks (rating), and Good Earning Capacity (ranking) and Good Financial Prospect (rating). For the German sample, these correlations were +0.67 and +0.54 , respectively, both significant beyond the 0.001 level. For the American sample, the correlations were +0.57 and +0.60 , respectively, both significant beyond the 0.001 level. In view of the fact that the analogous variables were worded slightly differently, consisted of single (and hence less than perfectly reliable) items, and appeared in the context of different sets of mate characteristics, these correlations can be viewed as reflecting rather high cross-method consistency of individual responses.

\section{DISCUSSION}

In this program of research, our goals were to examine similarities and differences in expressed mate selection criteria in males and females in the countries of Germany and the United States. To document these criteria, we conducted two studies using two methods applied to large samples-over $2500 \mathrm{Ss}$ participated in these studies. Our goals in this discussion are four: (1) to 
Table 6. Correlations between background

\begin{tabular}{|c|c|c|c|c|c|c|c|c|}
\hline \multirow[b]{3}{*}{ Mate characteristics } & \multicolumn{4}{|c|}{ Age } & \multicolumn{4}{|c|}{ Number of brothers } \\
\hline & \multicolumn{2}{|c|}{ German } & \multicolumn{2}{|c|}{ American } & \multicolumn{2}{|c|}{ German } & \multicolumn{2}{|c|}{ American } \\
\hline & $\mathbf{M}$ & $\mathbf{F}$ & $\mathbf{M}$ & $\mathbf{F}$ & $\mathbf{M}$ & $F$ & $\mathbf{M}$ & F \\
\hline Good Cook and Housekeeper & $33 * * *$ & 02 & -03 & -03 & 02 & -01 & $10^{*}$ & -01 \\
\hline Pleasing Disposition & $10^{*}$ & $11 *$ & -04 & $-09 *$ & -04 & -03 & -02 & -03 \\
\hline Sociability & 01 & -04 & -03 & -01 & 07 & 0I & -03 & -05 \\
\hline Similar Educational Background & $19 * * *$ & $16^{* * *}$ & -01 & $09^{*}$ & -01 & $-16 * * *$ & -07 & -01 \\
\hline Refinement, Neatness & -02 & $17 * * *$ & -05 & 04 & -03 & $-05^{* * *}$ & -02 & 01 \\
\hline Good Financial Prospect & 11 & $29 * * *$ & -04 & 03 & -07 & -02 & -04 & 01 \\
\hline \multicolumn{9}{|l|}{ Chastity (no previous sexual } \\
\hline intercourse) & $28 * * *$ & 06 & -06 & 00 & $11^{*}$ & 02 & -01 & 07 \\
\hline Dependable Character & $25 * * *$ & $18 * * *$ & -07 & -02 & 04 & 03 & -02 & 03 \\
\hline Emotional Stability and Maturity & $16 * * *$ & $22 * * *$ & -07 & 03 & 02 & $-02 * * *$ & -04 & 05 \\
\hline Desire for Home and Children & $34 * * *$ & $33 * * *$ & -03 & 02 & 06 & 02 & 04 & $11^{* *}$ \\
\hline Favorable Social Status or Rating & $24 * * *$ & $25^{* * *}$ & -05 & 07 & -04 & 04 & -09 & -01 \\
\hline Good Looks & $-13 * *$ & $-18 * * *$ & $-11 * *$ & -01 & 00 & -03 & -02 & 00 \\
\hline Similar Religious Background & $18 * * *$ & -09 & -06 & 03 & -03 & -01 & -00 & 06 \\
\hline Ambition and Industriousness & $19^{* * *}$ & $32 * * *$ & 00 & -03 & -03 & 03 & 00 & 02 \\
\hline Similar Political Background & 02 & 02 & $12^{* *}$ & 06 & -06 & $-10^{*}$ & -04 & -10 \\
\hline Mutual Attraction-Love & -06 & -02 & $-1 i^{* *}$ & -01 & -08 & 01 & 03 & 01 \\
\hline Good Health & $23 * * *$ & $17 * * *$ & $-09^{*}$ & 01 & 01 & 07 & 01 & 01 \\
\hline Education and Intelligence & 02 & $14 * * *$ & -01 & 00 & -07 & -03 & -02 & -03 \\
\hline
\end{tabular}

summarize the major findings that have emerged, (2) to discuss the theoretical significance of these findings, (3) to enumerate limitations of this research program, and (4) to identify directions for future research.

\section{Sex differences across cultures}

Two clusters of sex differences were predicted to occur across cultures. Females, more than males, were predicted to value in a potential mate the characteristic of good earning potential, as well as the features that often covary with earning potential such as ambition, industriousness, older age, and favorable social status. In contrast, males were predicted to value more than females the characteristics of youth and beauty. These sex differences were found in both countries in both studies using both methods. Indeed, sex differences on earning potential and physical attractiveness were the largest in magnitude to emerge within each of the two studies, the two countries, and two methods.

These results appear to support the hypothesis that males seek as mates those females whose reproductive value appears to be high, and that females seek as mates those males whose resources or resource potential appears to be high (cf. Buss, 1984, 1985, 1989; Buss and Barnes, 1986). They further suggest that these sex differences are not limited to the United States. Future studies could fruitfully examine the origins of these sex differences, whether in the socialization practices provided by parents or perhaps in genetic differences between males and females.

Additional sex differences emerged across cultures that were not explicitly predicted. Females, more than males, appear to value the mate characteristics of Similar Educational Background, Dependable Character, Emotional Stability and Maturity, Desire for Home and Children, and Education and Intelligence. In contrast, males more than females value Good Cook and Housekeeper and Chastity in potential mates. These findings, although not specifically predicted, are none the less consistent with an emphasis on the reproductive functions currently served by males and females.

Cross-cultural differences across the sexes. No specific predictions were made regarding differences between German and American mate selection values. Two differences emerged across both studies. Americans, more than Germans, appear to place greater emphasis on the physical attractiveness of a prospective mate. Germans, more than Americans, appear to place greater value on Good Housekeeping in a potential mate. This latter finding must be qualified; although it emerged from the ranking procedures in both Study I and II, it failed to emerge from the rating procedure for the slightly different item Good Cook and Housekeeper.

Several additional cultural differences emerged from Study II using the rating procedure. Germans, more than Americans, placed greater value on Dependable Character and Similar Political Background. Americans, more than Germans, placed greater value on Sociability, 
variables and mate preferences: ratings

\begin{tabular}{|c|c|c|c|c|c|c|c|c|c|c|c|}
\hline \multicolumn{4}{|c|}{ Number of sisters } & \multicolumn{4}{|c|}{ Age prefer to marry } & \multicolumn{4}{|c|}{ Number of children wanted } \\
\hline \multicolumn{2}{|c|}{ German } & \multicolumn{2}{|c|}{ American } & \multicolumn{2}{|c|}{ German } & \multicolumn{2}{|c|}{ American } & \multicolumn{2}{|c|}{ German } & \multicolumn{2}{|c|}{ American } \\
\hline $\mathbf{M}$ & $\mathbf{F}$ & $\mathbf{M}$ & $\mathbf{F}$ & M & $\mathbf{F}$ & M & $F$ & $\mathbf{M}$ & F & $\mathbf{M}$ & $\mathrm{F}$ \\
\hline 08 & 00 & 01 & 01 & -07 & 00 & -06 & $08^{*}$ & 08 & 05 & 07 & 06 \\
\hline-03 & -05 & -07 & -02 & -02 & 00 & -04 & 04 & 02 & -03 & 07 & -01 \\
\hline-08 & -03 & -01 & $-07^{* *}$ & $13^{*}$ & -04 & -06 & 01 & 05 & -02 & 00 & -01 \\
\hline 02 & -09 & -09 & -04 & 06 & $-10^{\circ}$ & $14 * * *$ & 08 & 02 & $10^{*}$ & -07 & -01 \\
\hline-07 & -09 & 02 & $-11^{* *}$ & -04 & 03 & 09 & 00 & -01 & -01 & 02 & 02 \\
\hline-06 & 06 & -05 & 01 & -01 & $-20^{* * *}$ & 06 & -04 & -02 & 02 & -07 & 01 \\
\hline $11 *$ & 02 & 07 & 08 & $-17 * *$ & $-11 *$ & $-24^{* * *}$ & $-17 * * *$ & $14^{* *}$ & $12^{*}$ & $14^{* * *}$ & $18 * * *$ \\
\hline-09 & 07 & 01 & 00 & 05 & $-12^{*}$ & -09 & -02 & 04 & $08^{*}$ & 01 & $09^{*}$ \\
\hline 04 & -08 & 00 & 02 & 01 & 04 & 07 & 01 & 05 & -02 & -03 & 08 \\
\hline 03 & 04 & 06 & 01 & $-26^{* * *}$ & $-32^{* * *}$ & $-25^{* * *}$ & $-33^{* * *}$ & $47^{* * *}$ & $40^{* * *}$ & $29 * * *$ & $39 * * *$ \\
\hline-04 & -06 & -07 & -03 & -05 & $-15^{* *}$ & -03 & -06 & $12^{*}$ & 05 & -04 & 02 \\
\hline 04 & $-17 * * *$ & 01 & -05 & $13^{*}$ & $10^{*}$ & $14^{* * *}$ & -10 & $-11^{*}$ & -04 & 01 & $-09^{*}$ \\
\hline 00 & 06 & 01 & 04 & -07 & -06 & $-20 * * *$ & $-24 * * *$ & 05 & $13^{*}$ & 04 & $17 * * *$ \\
\hline-03 & -03 & -01 & -02 & -08 & $-13^{* *}$ & 01 & -06 & 05 & 08 & -03 & 03 \\
\hline 06 & -06 & -02 & 00 & $13^{*}$ & $13^{* *}$ & 00 & 01 & -02 & -03 & 05 & -02 \\
\hline 01 & 06 & $\infty 0$ & -02 & 09 & $20^{* * *}$ & 00 & 01 & 02 & 01 & 03 & 03 \\
\hline-06 & 04 & -03 & 00 & 02 & -02 & $11^{* *}$ & -01 & 03 & -01 & -01 & 00 \\
\hline-03 & $12^{* *}$ & -04 & -06 & $11^{\circ}$ & -05 & $11^{* *}$ & $15^{* * *}$ & -01 & 06 & -06 & 01 \\
\hline
\end{tabular}

Refinement and Neatness, Emotional Stability, Desire for Home and Children, Favorable Social Status, Similar Religious Background, Ambition and Industriousness, and Chastity.

A few of these cultural differences can be partly attributed to the age differences between the German and American samples for Study II. For example, Dependable Character covaries positively with age for Germans, so their greater valuation of this characteristic may simply reflect the greater age of this sample. However, most of these findings can not be attributed to the significant age differences between the German and American samples in Study II. Indeed, age covaries positively within the German sample with valuation of characteristics such as Desire for Home, Emotional Stability and Maturity, Favorable Social Status, and Ambition and Industry. Thus, in spite of the fact that older German $S$ s value these characteristics more, and the German sample is older on average than the American sample in Study II, Americans none the less place greater value on this set of mate characteristics.

Size of family of origin and children desired. The most important correlates of mate preferences center around size of family. Across both cultures, those who come from large families indicate that they want more children than do those who come from smaller families. In addition, across both cultures, those who plan to marry later in life desire fewer children than do those who plan to marry early. Within Germans, there were additional correlates of family size preference: the portrait of the $S$ who wants many children is one who plans to marry early, places religiosity high on mate preference values, does not care as much for a physically attractive mate, and comes from a large family. Future research could fruitfully examine whether this cluster of early marriage, large family of origin, large number of children desired, high religiosity, and de-emphasis on physical attractiveness coheres within other cultures.

Limitations and future directions. This research has several limitations that could be profitably addressed in future studies. Perhaps the most potent limitation is that all data were derived from a single source-self report. On the assumption that mate values are sometimes discussed with, and observed by, friends and family members, these kin and non-kin relations could be used as additional data sources to obtain mate selection criteria.

A second limitation concerns the links between expressed mate selection criteria and actual mating decisions. There is some evidence within the United States, for example, that physically attractive females and males high on income and occupation status tend to mate with one another (Elder, 1969; Taylor and Glenn, 1976). This finding provides support for the corresponding sex differences obtained in the present studies using expressed criteria. Analogous studies should be conducted in Germany to corroborate this link between expressed criteria and actual mating decisions. None the less, the links are likely to be complex. The characteristics of an obtained mate are likely to be some function of (a) expressed mate selection criteria, (b) the pool of eligible mates, and (c) the desirability of the mates one can command given one's personal attributes, resources, 
and powers of persuasion. Not all individuals will be successful in obtaining intelligent, attractive, industrious, dependable, kind, understanding, high-income partners, in spite of their expressed preferences.

A third limitation is that only two cultures were used in these studies, and some might argue that these cultures are remarkably similar to each other. Thus, the next crucial step in this research program is to examine a wider variety of cultures from around the world. Such a study is currently underway (Buss, 1989). This research should be capable of addressing questions such as: Do Americans stand out in the high value placed on physical appearance? Are Germans unique among cultures in their emphasis on good housekeeping and dependability in potential mates? And are sex differences in mate selection criteria universal?

\section{REFERENCES}

Berscheid E. and Walster E. (1974) Physical attractiveness. In Advances in Experimental Social Psycholngy (Edited by Berkowitz L.), pp. 157-215. Academic Press, New York.

Burgess E. W. and Wallin P. (1953) Engagement and Marriage. Lippincott, Philadelphia.

Buss D. M. (1984) Evolutionary biology and personality psychology: toward a conception of human nature and individual differences. Am. Psychol. 39, 1135-2247.

Buss D. M. (1985) Human mate selection. Am. Sci. 73, 47-51.

Buss D. M. (1987) Sex differences in human mate selection criteria: an evolutionary perspective. In Sociobiology and Psychology: Issues, Ideas and Application (Edited by Crawford C., Smith M. and Krebs D.). Erlbaum, Hillsdale, N.J.

Buss D. M. (1989) Sex differences in human mate preferences: evolutionary hypotheses tested in 37 cultures. Behat. Brain Sci. 12, 1-49.

Buss D. M. and Barnes M. (1986) Preferences in human mate selection. J. Person. Soc. Psychol. 50, 559-570.

Christensen H. (1979) Attitudes toward marital infidelity: a nine-culture sampling of university student opinion. In Cross-Cultural Perspectives of Mate-Selection and Marriage (Edited by Kurian G.). Greenwood Press, Westport, Conn.

Crow J. F. and Kimura M. (1970) An Introduction to Population Genetics Theory. Harper \& Row, New York.

Darwin C. (1871) The Descent of Man and Selection in Relation to Sex. Murray, London.

Eckland B. K. (1968) Theories of mate selection. Eugen. Q. 15, 71-84.

Elder G. H. Jr (1969) Appearance and education in marriage mobility. Am. Soc. Rev. 34, 519-533.

Fisher R. A. (1958) The Genetical Theory of Natural Selection. Clarendon Press, Oxford.

Gough H. G. (1973) Personality assessment and the study of population. In Psychological Perspectives on Population (Edited by Fawcett J. T.), pp. 329-353. Basic Books, New York.

Hill R. (1945) Campus values in mate selection. J. Home Econ. 37, 554-558.

Hudson J. W. and Henze L. P. (1969) Campus values in mate selection: a replication. J. Marr. Fam. 31, 772-775.

Jensen A. R. (1978) Genetic and behavioral effects of nonrandom mating. In Human Variation: Biopsychology of Age, Race and Sex (Edited by Osborne R. T., Noble C. E. and Wey N. J.), pp. 51-109. Academic Press, New York.

Langhorne M. C. and Secord P. F. (1955) Variations in marital needs with age, sex, marital status, and regional composition J. Soc. Psychol. 41, 19-37.

McGinnis R. (1958) Campus values in mate selection. Soc. Forces 36, 368-373.

Murstein B. I. (1979) Qualities of desired spouse: a cross-cultural comparison between French and American College students. In Cross-Cultural Perspectives of Mate-Selection and Marriage (Edited by Kurian G.). Greenwood, Westport, Conn.

Podmore D. and Chaney D. (1979) Attitudes toward marriage and the family amongst young people in Hong Kong, and Comparisons with the United States and Taiwan. In Cross-Cultural Perspectives of Mate-Selection and Marriage (Edited by Kurian G.). Greenwood, Westport, Conn.

Raschke V. and Li A. (1979) Premarital sexual permissiveness of college students in Hong Kong. In Cross-Cultural Perspectives of Mate-Selection and Marriage (Edited by Kurian G.). Greenwood, Westport, Conn.

Rushton J. P. (1984) Genetic similarity theory: beyond kin selection. Behav. Genet. 14, 179-193.

Symons D. (1979) The Evolution of Human Sexuality. Oxford University Press, New York.

Taylor P. A. and Glenn N. D. (1976) The utility of education and attractiveness for females' status attainment through marriage. Am. Soc. Rev. 41, 484-498.

Thiessen D. D. and Gregg B. (1980) Human assortative equilibrium and genetic equilibrium: an evolutionary perspective. Ethol. Sociobiol. 1, 111-140.

Trivers R. (1972) Parental investment and sexual selection. In Sexual Selection and the Descent of Man: 1871-1971 (Edited by Campbell B.), pp. 136-179. Aldine, Chicago.

Vandenberg S. G. (1972) Assortative mating. or who marries whom? Behav. Genet. 2, 127-158. 